\title{
Influencia de los Sistemas de Gestión Ambiental ISO 14001 en las organizaciones: caso estudio empresas manufactureras de Barranquilla
}

\author{
Influence of environmental management systems in organizations ISO 14001: \\ case study manufacturing enterprises of Barranquilla
}

\author{
Norberto Acuña ${ }^{1} \quad$ Lindsay Figueroa $^{1} \quad$ María Jimena Wilches $^{1 *}$ \\ Recibido 16 de diciembre de 2014, aceptado 14 de enero de 2016 \\ Received: December 16, 2014 Accepted: January 14, 2016
}

\begin{abstract}
RESUMEN
El Sistema de Gestión Ambiental ISO 14001 tiene como objetivo servir de soporte a la prevención y manejo de la contaminación ambiental, en equilibrio con las necesidades socioeconómicas de los diferentes sectores de la sociedad.

En un grupo de 13 empresas certificadas por el Instituto colombiano de Normas Técnicas "ICONTEC" en la ciudad de Barranquilla se realizó un diagnostico por medio de encuestas a empresarios, para determinar las ventajas y desventajas de la aplicación del Sistema de Gestión Ambiental en términos de la gestión organizacional, gestión financiera, relaciones comerciales y el aprovechamiento de los recursos, con el fin de formular estrategias de sensibilización al sector empresarial de los beneficios identificados y en coherencia con las características de la región estudiada.

Por medio de esta investigación se identificaron los beneficios de la implementación de este sistema administrativo, orientado por la planeación estratégica de la organización en la rentabilidad de la empresa, como lo son una mejor imagen corporativa, un mejor desempeño jurídico, un adecuado manejo de los recursos, la reducción en la generación de residuos y la atracción de nuevos clientes; ratificándose este como una herramienta importante de la administración actual y vital para la generación de ventajas competitivas.

Se espera que los resultados de esta investigación motiven al sector manufacturero en la búsqueda de certificar sus procesos en gestión ambiental y que les permita lograr mayor competitividad.
\end{abstract}

Palabras clave: Sistemas de gestión, impacto ambiental, gestión financiera, gestión organizacional, aprovechamiento de recursos, relaciones comerciales.

\begin{abstract}
Environmental Management System ISO 14001 aims to provide support for the prevention and management of environmental pollution in balance with socio-economic needs of the different sectors of society. In a group of 13 ICONTEC certified companies from the City of Barranquilla performed a diagnosis through business surveys that were conducted to determine the advantages and disadvantages of implementing Environmental Management System in terms of organizational management, financial management, business relationships and the use of resources in order to develop strategies to the business sector awareness of the benefits identified and consistent with the characteristics of the studied region.
\end{abstract}

\footnotetext{
1 Departamento de Ingeniería Industrial. Universidad Autónoma del Caribe. Calle 90 \# 46-112. Barranquilla, Colombia. E-mail: mwilches@uac.edu.co; nacuna@uac.edu.co; lindsay.figueroa@uac.edu.co

* Autor de correspondencia
} 
Through this research the benefits of implementing this administrative system, guided by the strategic planning of the organization in the profitability of the company were identified, as they are a better corporate image, a better legal performance, adequate management resources, reduced waste generation and attracting new customers; ratifying it as an important tool of the present administration and for the generation of competitive advantages.

It is expected that the results of this research would motivate the manufacturing sector in seeking to certify their environmental management and processes that will achieve greater competitiveness.

Keywords: Management systems, environmental impact, financial management, organizational management, resources use, trade relations.

\section{INTRODUCCIÓN}

Teniendo en cuenta el libre comercio internacional y la intención de implementar estrategias de fortalecimiento empresarial por medio del desarrollo sostenible, las empresas en el distrito de Barranquilla desconocen como el Sistema de Gestión Ambiental ISO 14001-2004 puede servir como una herramienta administrativa para el logro de ventajas competitivas, lo que comúnmente se cree solo puede ser logrado por medio de un Sistema de Gestión de Calidad ISO 9001.

La mayoría de las empresas se han visto interesadas en implementar ISO 14001-2004 sin desarrollar un análisis profundo de los efectos que esta genera sobre los aspectos administrativos de la organización y en muchos casos no se tiene en cuenta una planeación estratégica adecuada. Por lo tanto la aplicación del Sistema de Gestión, frecuentemente es orientada a obtener una certificación que permita acceder al mercado, eliminando barreras competitivas menospreciando la prerrogativa de esta herramienta administrativa.

Es importante recalcar para esta investigación el buen momento de desarrollo económico de la ciudad y el departamento, esto a partir de las ventajas competitivas de los tratados de libre comercio firmados por el país y una acertada política de administración pública local. La ciudad se posiciona como un epicentro en materia de atracción de inversiones a nivel nacional e internacional, por lo que es importante revisar de qué manera se pueden aplicar estrategias de gestión ambiental que permitan acceder a nuevos mercados.

En esta investigación se pretende identificar los beneficios de la aplicación del Sistema de
Gestión Ambiental en términos de crecimiento rentable del negocio como iniciativa para adquirir mayor participación en el mercado, evaluando el impacto de la implementación de ISO 14001 en empresas certificadas por ICONTEC en la ciudad de Barranquilla para determinar sus ventajas y desventajas.

\section{ANÁLISIS TEÓRICO}

El progreso, consecuencia inobjetable de la industrialización, ha generado crecimiento poblacional y conductas de consumo excesivas lo que ha creado enorme presión sobre el ambiente y los recursos naturales en que se sustenta la producción.

La preocupación por los problemas ambientales se hizo evidente a mediados del siglo XX, como consecuencia de la contaminación provocada por el acelerado desarrollo industrial. Comenzó entonces a difundirse una serie de ideas que cuestionaban el modelo de crecimiento económico imperante y sus implicaciones en la degradación del ambiente y la afectación de los recursos naturales [1-2].

Si bien es cierto, las organizaciones no actúan de manera correcta sobre los factores ambientales, tanto así que en su formulación estratégica muchas veces no direccionan actividades para ello, centrándose solo en rebajar los costos y riesgos que puedan repercutir en sanciones, en inversiones y reparaciones económicas en sentido ambiental no viéndolo como una oportunidad de negocio, como lo explica quienes sustentan que "El Consumo y Producción Sustentable (CPS) no es una posibilidad u oportunidad, sino una necesidad" dadas las condiciones medioambientales actuales [3]. 
Estamos actualmente inmersos en un proceso de cambio. Este proceso comenzó realmente con la aparición del concepto de "desarrollo sostenible" y su aceptación prácticamente universal en la Conferencia de Río de 1992. Dicho desarrollo sostenible se basa en el llamado triple bottom, es decir, en la visión conjunta e integrada de los factores económicos, sociales y ambientales que afectan a las actividades productivas. Este nuevo enfoque ha modificado lenta pero profundamente la influencia de determinados factores ambientales: la gestión de los residuos, el uso de determinados materiales y, especialmente, todo el entorno de la energía relacionado con el Protocolo de Kioto. De manera inmediata se establecerán nuevas prioridades ambientales, que previsiblemente estarán especialmente relacionadas con la construcción y el transporte. Parece evidente que las profundas modificaciones de estas variables reclamarán enfoques estratégicos por parte de las empresas [4].

Entre las principales estrategias propuestas a nivel mundial para enfrentar los problemas ambientales se encuentran los Sistemas de Gestión Ambiental (SGA), creados como rutas para identificar y manejar sistemáticamente los aspectos e impactos ambientales generados por parte de las empresas. Los SGA están estrechamente relacionados con los sistemas de gestión de la calidad, en este sentido un SGA le permite a la empresa controlar los procesos susceptibles de generar daños al medio ambiente, minimizando los impactos ambientales de sus operaciones y mejorar el rendimiento de sus procesos.

La implementación de los SGA, mejora la actuación ambiental y las metas económicas trazadas por la organización, pues se enfoca en la búsqueda de un desarrollo sostenible bajo un esquema ecoeficiente, aplicado a todos los procesos productivos. Teniendo en cuenta lo anterior, solo aquellas organizaciones cuya dirección se encuentran comprometida con los objetivos planteados en su política, asigna recursos adecuados para poder implementar las medidas ambientales necesarias, comunicación y entrenamiento de los trabajadores de la empresa y auditorías integradas para poder realizar un seguimiento de los aspectos ambientales; han logrado alcanzar la excelencia ambiental en el funcionamiento y la aplicación de los SGA propuestos [5].
Los aspectos ambientales no deben ser responsabilidad únicamente del departamento de medio ambiente, sino que deben ser abordados por todas las áreas de la organización, incluyendo a los proveedores [6].

Actualmente la Norma ISO 14001 define el sistema de gestión como un grupo de elementos interrelacionados usados para establecer la política y los objetivos, así como para su cumplimiento. Además, dichos sistema de gestión debe incluir la estructura de la organización, la planificación de las actividades, las responsabilidades, prácticas, los procedimientos, los procesos y los recursos necesarios.

De acuerdo con lo anterior, un SGA es la integración armónica de los elementos requeridos para desarrollar una gestión enfocada en prevenir la contaminación, cumplir los requisitos y la legislación ambiental, y mejorar continuamente el desempeño ambiental. Por tanto, pretende desarrollar e implementar la política ambiental de la organización, y gestionar sus aspectos ambientales, a fin de servir de soporte a la prevención y manejo de la contaminación ambiental, en equilibrio con las necesidades socioeconómicas de los diferentes sectores de la sociedad [7].

Una vez implementado el sistema de gestión afectará a todos los aspectos de la administración de una organización en sus responsabilidades ambientales y ayudará a las empresas a tratar sistemáticamente dichos asuntos, con el fin de mejorar el comportamiento ambiental y las oportunidades de beneficio económico. Los estándares son voluntarios, no tienen obligación legal y no establecen un conjunto de metas cuantitativas en cuanto a niveles de emisiones o métodos específicos de medir esas emisiones.

Los principales objetivos de un SGA:

- Identificar los aspectos ambientales significativos, al igual que la legislación aplicable y otros requisitos.

- Formular y asumir el compromiso de política ambiental.

- Concretar el compromiso con objetivos, metas y acciones específicas.

- Lograr los objetivos y metas previstas.

En la actualidad, los empresarios y las autoridades se centran en el costo material de eliminar o reducir la contaminación. Sin fijarse en los costos de 
oportunidad (los recursos malgastados, el trabajo malgastado y la pérdida de valor del producto). En el plano de la productividad de los recursos hay que tener en cuenta que la mejora medioambiental y la competitividad van de la mano.

Debemos tener en cuenta que si una empresa contamina, está malgastando sus recursos, fallando en diseños o teniendo ineficiencia en el proceso de fabricación [8].

En las siguientes tablas se detallan los beneficios de la implementación del SGA ISO - 14001 tanto para los procesos como para los productos.

Tabla 1. Beneficios del Sistema de Gestión Ambiental ISO-14001 para los procesos.

\begin{tabular}{|l|l|}
\hline \multicolumn{2}{|c|}{ Beneficios para los procesos } \\
\hline 1. & $\begin{array}{l}\text { Ahorro de material por tratamiento completo, } \\
\text { por sustitución, por reutilización o por reciclado } \\
\text { de los materiales de producción }\end{array}$ \\
\hline 2. & Aumento de la productividad de los procesos \\
\hline 3. & $\begin{array}{l}\text { Menos paradas gracias a un control y un man- } \\
\text { tenimiento más cuidadosos }\end{array}$ \\
\hline 4. & Mejor utilización de los subproductos \\
\hline 5. & Conversión de los residuos en materias valiosas \\
\hline 6. & $\begin{array}{l}\text { Menos consumo de energía durante el proceso } \\
\text { de producción }\end{array}$ \\
\hline 7. & $\begin{array}{l}\text { Reducción de los costos de almacenamiento y } \\
\text { transporte de los materiales }\end{array}$ \\
\hline 8. & $\begin{array}{l}\text { Ahorros gracias a una mayor seguridad en el } \\
\text { trabajo }\end{array}$ \\
\hline 9 & $\begin{array}{l}\text { Supresión o reducción del costo de las actividades } \\
\text { necesarias para la manipulación, transporte y } \\
\text { eliminación de desechos y residuos }\end{array}$ \\
\hline 10. & $\begin{array}{l}\text { Mejoras en el producto debido a los adelantos } \\
\text { del proceso }\end{array}$ \\
\hline
\end{tabular}

Fuente: Ser Competitivo. Porter, Michael. 2003.

Tomando como referencia lo anterior, los sistemas de gestión y las certificaciones se han convertido en una parte imprescindible en la organización y en un requisito para la supervivencia en el siglo XXI [10], pero no todas las organizaciones han alcanzado el mismo nivel de madurez en SGA, algunas contemplan la gestión ambiental como un instrumento para mejorar la eficiencia de sus procesos, al mismo tiempo que cuidan el medio ambiente [11] y muchas otras se limitan a cumplir los requisitos de
Tabla 2. Beneficios del Sistema de Gestión Ambiental ISO-14001 para los productos.

\begin{tabular}{|c|l|}
\hline \multicolumn{2}{|c|}{ Beneficios de los productos } \\
\hline 1. & Productos de mayor calidad y más uniformes \\
\hline 2. & $\begin{array}{l}\text { Reducción del costo del producto (sustitución } \\
\text { de materiales) }\end{array}$ \\
\hline 3. & Menos costos de embalaje \\
\hline 4. & $\begin{array}{l}\text { Productos que utilizan más eficazmente los } \\
\text { recursos }\end{array}$ \\
\hline 5. & \\
\hline 6. & Productos más seguros \\
\hline 7. & $\begin{array}{l}\text { Menos costos netos de eliminación del producto } \\
\text { para el usuario }\end{array}$ \\
\hline 8. & Mayor valor residual y de reventa del producto \\
\hline
\end{tabular}

Fuente: Ser Competitivo. Porter, Michael 2003 [9].

la legislación. En efecto, las pequeñas y medianas empresas que no buscan colocar sus productos en mercados externos, optan por implantar un SGA que les permita cumplir con la normativa legal vigente en sus países de operación. Las empresas grandes, por el contrario, suelen requerir la certificación ISO 14001 para competir exitosamente en mercados más exigentes [12].

La gestión ambiental es un medio para llegar a los consumidores finales utilizando el marketing verde y el mercadeo ecológico para socializar y divulgar los avances de las organizaciones en el ámbito ambiental, estos son los resultados de una producción más limpia por medio del uso de nuevas tecnologías que contaminen menos, lo que se traduce en un menor impacto ambiental, menor consumo de recursos naturales, menores desperdicios y mayor productividad, atrayendo así a los consumidores que se inician en el desarrollo de una cultura ecológica e interesados en la protección del medio ambiente, el marketing verde cumple una doble finalidad atraer nuevos clientes y fidelizar a los antiguos y generar conciencia y educación ambiental a todos sus grupos interesados [13].

La aplicación del principio del triple bottom (económico, ambiental y social) está modificando los criterios utilizados para la valoración de empresas. A los clásicos conceptos de valoración de activos y de recursos financieros, se añaden actualmente los de marca y reputación, de capital humano e intelectual, y donde junto a inversores y clientes se encuentran los empleados de la compañía, los 
miembros de la comunidad en la que la empresa ejerce sus actividades, y las firmas comerciales relacionadas con la empresa. En síntesis, y por coherencia con el principio del triple bottom, a los criterios económicos se añaden los sociales y ambientales en el momento de configurar el valor real de la empresa. Estos nuevos criterios de valoración son especialmente visibles en la cotización en bolsa y en el momento de una transacción de compra-venta [14].

La influencia de las variables ambientales en la valoración de las empresas, viene reflejada lógicamente en su valoración en bolsa. A los estrictos criterios económicos se unen los sociales y ambientales; estos últimos tanto por modificación de normas legales, como por una cierta reorientación de la demanda, ya que muchos inversores desean que sus fondos sean destinados a empresas que, además de beneficios económicos, aporten respeto social y ambiental.

El perfil productivo de la ciudad se establece con información del fondo de promoción para el desarrollo de la ciudad, denominado ProBarranquilla [15], allí se presenta una clasificación de la participación de los subsectores industriales en las exportaciones.

Para la toma de datos se aplicó una encuesta tipo Lickert, enfocada al personal directivo, en ella se valoraron aspectos de gestión, financieros, comerciales y aprovechamiento de recursos naturales, el tipo de preguntas formuladas se ajustaron según las características de competitividad de Porter [9] la muestra tomada para la investigación es de 13 empresas y se conservan los nombres por requerimientos de confidencialidad.

Se realizó el análisis de la encuesta y se caracterizaron las variables de mayor influencia sobre los SGA, clasificadas en Gestión Organizacional, gestión financiera, gestión comercial y aprovechamiento de recursos. De acuerdo a los resultados de este análisis se proyectaron las estrategias a partir de las que se pretende potencializar los beneficios de los SGA.

Para lograr la investigación planteada se muestra la Figura 1 que resume la metodología desarrollada para determinar la influencia de los SGA en las organizaciones manufactureras en Barranquilla.

\section{METODOLOGÍA}

Para determinar la influencia de los SGA en las organizaciones, tomando como caso de estudio las empresas manufactureras de la ciudad de Barranquilla se desarrolló una investigación exploratoria que permitiera identificar las variables que inciden directamente sobre la actividad industrial. Para esto se recurrió a los encargados de los procesos de certificación de las empresas y mediante una encuesta establecer la percepción y la experiencia resultante de los logros alcanzados.

\section{RESULTADOS}

\section{Análisis de los componentes organizacionales}

En el departamento del Atlántico, durante el período 2012-2013 se ha mantenido un ritmo de crecimiento anual del número de sociedades constituidas de $7 \%$, esto se refleja en la permanente reducción de los índices de desempleo ocupando los primeros lugares en este indicador a nivel nacional. Los

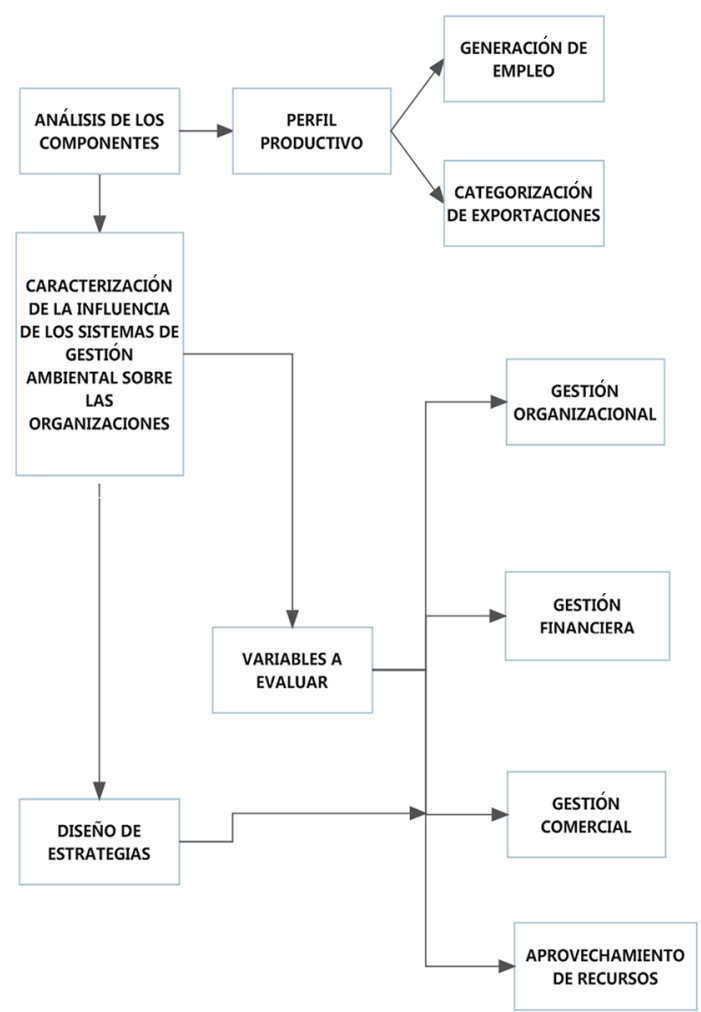

Figura 1. Metodología de la investigación sobre la influencia de los SGA en las organizaciones en Barranquilla. 
productos industriales de Barranquilla con potencial para exportar, se destacan los sectores de alimentos y bebidas, confecciones y calzado, textil, cueros, maderas, manufacturas, maquinaria y equipo y otros productos metálicos, productos de minerales no metálicos, plástico y caucho y químicos.

Las exportaciones del departamento del Atlántico están asociadas a la producción industrial; mientras que el $75,1 \%$ de las exportaciones no tradicionales del país son productos industriales, en Atlántico lo son el 95,7\%, información que recalca la vocación de puerto exportador [16]. Las exportaciones del Atlántico entre 2011 y 2012 fueron en su mayoría industriales, sobresaliendo las manufacturas e insumos básicos y derivados, seguido de la agroindustria.

\section{Categorización de las empresas según el sector productivo}

Según sectores económicos, en el año 2013 las exportaciones del Atlántico continuaron lideradas por la industria manufacturera, representando el $83,3 \%$ del valor global, es decir, US\$ 1.206 millones FOB, seguida por el comercio con el $13,8 \%$. La Figura 2 muestra la distribución de sectorial de las exportaciones al año 2013.

Dentro de la industria manufacturera se destacó la comercialización de productos químicos con una participación del 48,2\%, o sea, US\$ 606 millones FOB del total industrial, seguidos de alimentos y bebidas con el 10,5\% y metalmecánica con el 10,3\%; en conjunto, en estos subsectores se concentró

\section{DISTRIBUCIÓN SECTORIAL DE LAS} EXPORTACIONES 2013

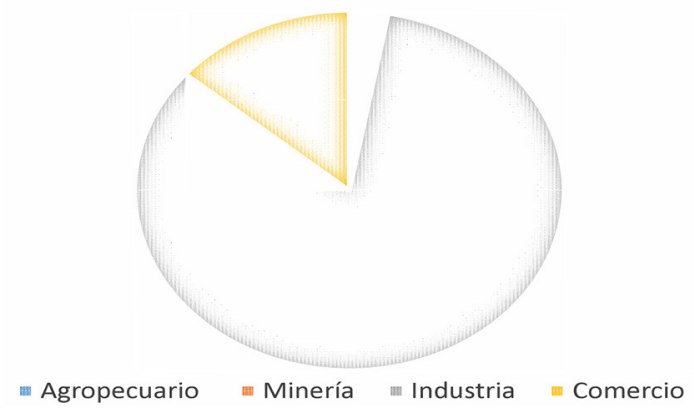

Figura 2. Distribución sectorial de las exportaciones 2013.

Fuente: Los autores. Adaptado de [16]. el $69 \%$ del valor total exportado por la actividad industrial en el 2013. Se determina entonces el perfil de las empresas exportadoras del departamento del Atlántico en donde sobresale el liderazgo de las empresas del sector químico, como se observa en la Figura 3.

Para el desarrollo del análisis de las variables se recurrió a una muestra de trece empresas localizadas en la ciudad de Barranquilla, asociadas a este perfil, con el objeto de establecer la relación entre las características del entorno y los efectos de los sistemas de gestión ambiental en ellas.

\section{Caracterización de la influencia}

En esta fase se realizó un análisis de las variables de gestión organizacional, gestión financiera, gestión comercial y el aprovechamiento de los recursos por medio de la aplicación de una encuesta en empresas exportadoras que cuentan con un sistema de gestión ambiental.

En cuanto a la gestión organizacional se pretendió establecer la integración del Sistema de Gestión Ambiental con gestión empresarial y otros factores importantes como lo son la imagen corporativa y los procesos de comunicación interna.

Con respecto a la gestión financiera se evaluaron aspectos como la inversión requerida para la implementación y sostenimiento de sistema, la vinculación entre los indicadores financieros y ambientales, los beneficios tributarios y la minimización de costos asociados a la gestión de residuos.

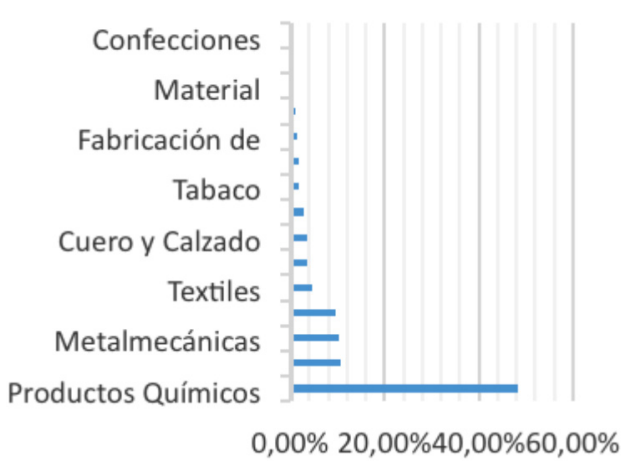

Figura 3. Perfil de los sectores empresariales exportadores del departamento del Atlántico. Fuente: los autores: adaptado de [17] . 
En la variable de gestión comercial se evaluó los beneficios de la implementación en estrategias comerciales y apertura de nuevos mercados.

Por último, se analizó en el aprovechamiento de recursos se observaron la reconversión tecnológica de procesos y la reducción en la generación de residuos.

Tabla 3. Variables analizadas en la investigación.

\begin{tabular}{|l|l|}
\hline \multicolumn{1}{|c|}{ Variables } & \multicolumn{1}{c|}{ Aspecto a evaluar } \\
\hline $\begin{array}{l}\text { Gestión } \\
\text { organizacional }\end{array}$ & $\begin{array}{l}\text { Compromiso de la Dirección } \\
\text { Plan de Comunicación Interna Imagen } \\
\text { Corporativa } \\
\text { Objetivos y experiencia ambiental }\end{array}$ \\
\hline $\begin{array}{l}\text { Gestión } \\
\text { financiera }\end{array}$ & $\begin{array}{l}\text { Inversión ambiental } \\
\text { Indicadores financieros-ambientales } \\
\text { Costos por unidad de recurso } \\
\text { Beneficios tributarios }\end{array}$ \\
\hline $\begin{array}{l}\text { Gestión } \\
\text { comercial }\end{array}$ & $\begin{array}{l}\text { Tasa de crecimiento de nuevos mercados } \\
\text { Relación con proveedores } \\
\text { Relación con los clientes } \\
\text { Impacto en nuevos mercados }\end{array}$ \\
\hline $\begin{array}{l}\text { Reconversión tecnología de procesos } \\
\text { de recursos }\end{array}$ & $\begin{array}{l}\text { Clasificación de residuos sólidos } \\
\text { Manejo de la contaminación } \\
\text { Reducción en la generación de residuos } \\
\text { Manejo de los recursos } \\
\text { Experiencias de ahorro energético }\end{array}$ \\
\hline
\end{tabular}

Fuente: Los autores.

Una vez aplicadas las encuestas se obtuvieron de cada variable estudiada los resultados según la Figura 4:

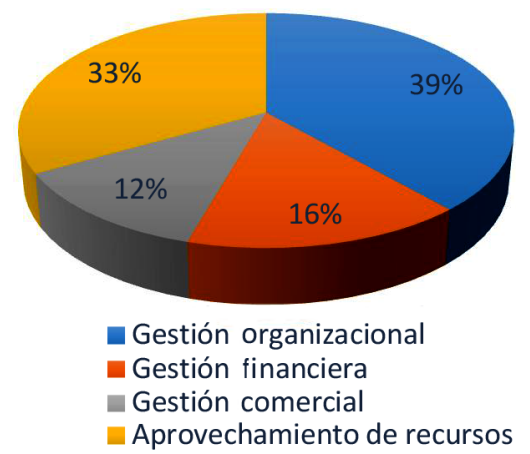

Figura 4. Área de la empresa con mayor impacto del Sistema de Gestión Ambiental.
Se evidencia que la implementación del SGA tiene mayor impacto en la gestión organizacional, seguida por el aprovechamiento de los recursos. Por su parte, la gestión financiera y gestión comercial son poco influenciadas.

De acuerdo a la Figura 5, el desempeño jurídico es el factor de mayor relevancia de acuerdo con la percepción de los empresarios, esto debido a la obligatoriedad del cumplimiento de los estándares legales en todo proceso de certificación de la gestión ambiental. Con respecto a la imagen pública los empresarios la valoraron significativamente como resultado de la cultura ambiental que ha surgido en el entorno teniendo en cuenta la presión social a partir los problemas ambientales que se han presentado durante las últimas décadas y que se espera se potencialicen si no hay adecuado manejo dentro de los procesos productivos.
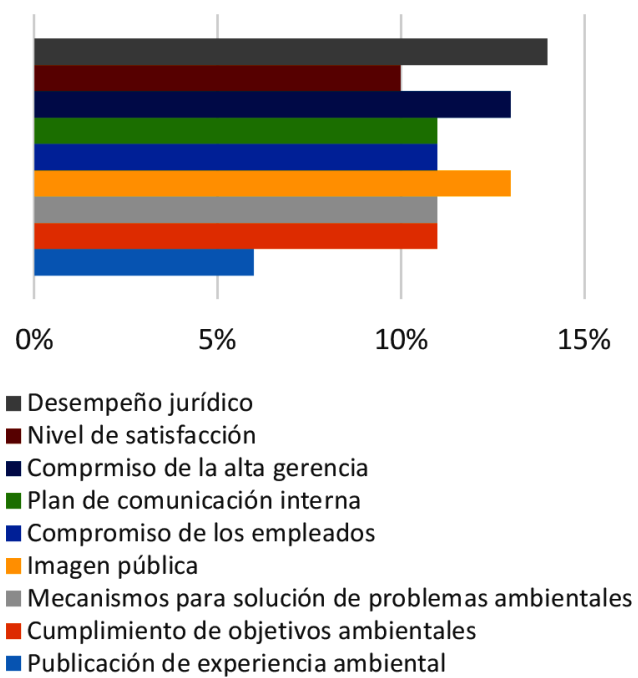

Figura 5. Incidencia de los SGA sobre las variables de gestión organizacional.

Como resultado de la gestión ambiental, según la Figura 6, el ahorro por mejoras ambientales es considerado un factor que genera beneficios sobre la gestión financiera asociado a las actividades de mejora que se desarrollan en los procesos, como: el reúso del agua, el uso eficiente de la energía, la reducción de consumo de materia prima, lo que a su vez genera impacto sobre la generación de residuos. Este mismo factor se relaciona con los resultados obtenidos en el cumplimiento de indicadores y la contabilidad de costos por unidad de recursos. 


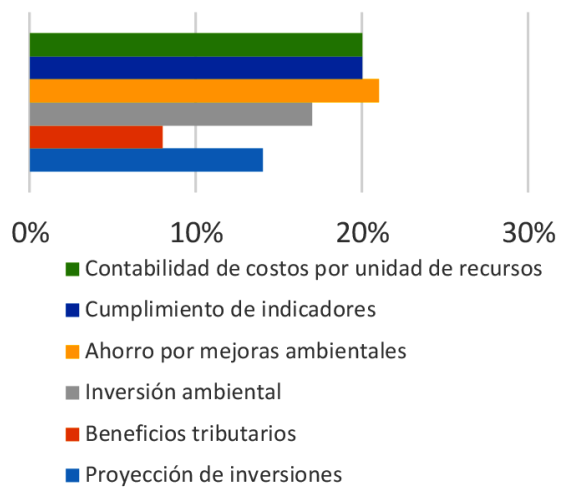

Figura 6. Incidencia de los SGA sobre las variables de gestión financiera.

Por otro lado, se evidencia una percepción débil en cuanto a los beneficios tributarios generados por la implementación del sistema, debido a que en el país no existen políticas claras de reducción de impuestos por la aplicación de buenas prácticas ambientales. Es decir, actualmente no existen reconocimientos por prevenir sino que se paga por contaminar.

De acuerdo con los empresarios, la gestión comercial se ve favorecida en la captación de nuevos clientes y el impacto en nuevos mercados precisamente por la buena imagen que genera para la sociedad el saber que las empresas tienen un compromiso con el medio ambiente y la garantía de procesos limpios, ver Figura 7.

De acuerdo a la Figura 8, con los empresarios el principal pilar de la gestión ambiental es la minimización de los residuos generados por los procesos y los programas de mejora para manejo

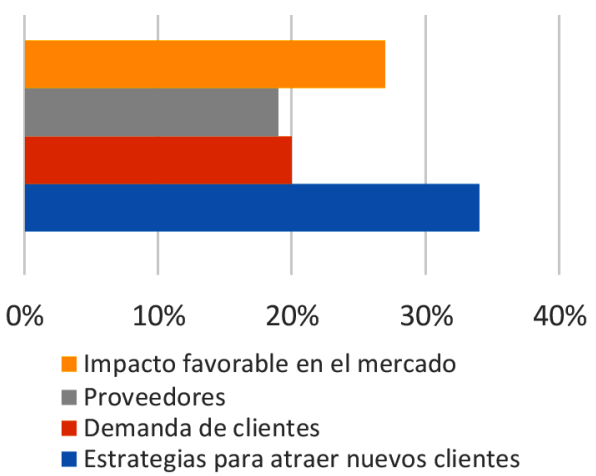

Figura 7. Incidencia de los SGA sobre las variables de gestión comercial.

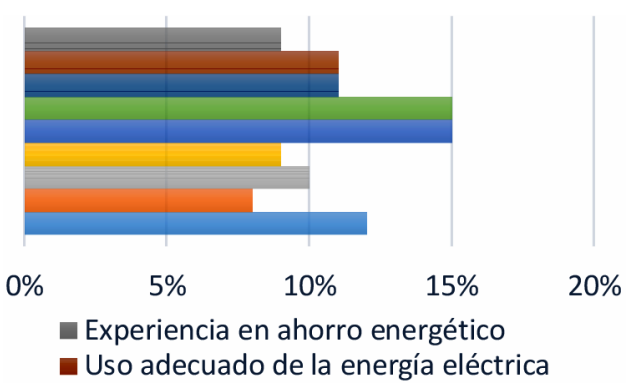

Figura 8. Incidencia de los SGA sobre las variables de Aprovechamiento de recursos.

de recursos. Estos programas se hacen de manera sistemática aprovechando los lineamientos de los SGA bajo la Norma ISO 14001.

La valoración más baja estuvo reflejada en el factor de uso adecuado de la energía eléctrica debido a que las empresas deben llegar a un nivel de madurez del sistema de gestión para lograr formular programas tendientes al buen uso de la energía eléctrica u otras fuentes de energía. Las organizaciones inician resolviendo problemas básicos y con el tiempo van escalando a niveles más exigentes.

\section{Estrategias de la influencia de la implementación de sistemas de gestión ambiental en la competitividad de las empresas}

De acuerdo con la caracterización efectuada se observa que las empresas certificadas con el SGA ISO 1400104 presentan un aprovechamiento apenas aceptable de los beneficios y ventajas competitivas que genera esta norma. Por tanto, se propusieron una serie de estrategias, teniendo en cuenta cada uno de las variables analizadas (gestión organizacional, gestión financiera, gestión comercial y el aprovechamiento de recursos), a fin de sensibilizar al empresario para que incorpore prácticas que lo lleven al aprovechamiento máximo de los beneficios generados por la implementación de un SGA.

\section{Estrategias de gestión organizacional}

- Planificar e impulsar proyectos de investigación, encaminados a conocer los beneficios que genera la implementación del SGA, o en su defecto, la incorporación de nuevas prácticas en pro del desarrollo sostenible.

- Resaltar las nuevas prácticas tecnológicas generadas para beneficio del medio ambiente, con la ayuda de los medios de comunicación y 
las redes sociales, con el fin de mejorar la imagen pública de las organizaciones, punto vital a la hora de poner en marcha estrategias comerciales.

- Establecer programas de control y auditorías internas que permitan dar seguimiento a la gestión ambiental aplicada, detectar falencias y tomar las medidas correctivas pertinentes.

- Elaborar un plan de comunicación que permita a las empresas un contacto directo con la comunidad y una retroalimentación en cuanto a aspectos medioambientales.

- Evaluar los resultados de los indicadores de gestión de los procesos ambientales que se lleven a cabo en las empresas, y hacer el respectivo plan de mejoramiento ambiental.

- Implementar políticas y programas de responsabilidad ambiental y tecnologías limpias.

- Revisar constantemente las actualizaciones reglamentarias y de normatividad asociados a los impactos ambientales generados por la actividad económica de la empresa a fin de hacer los ajustes necesarios cuando corresponda.

- Desarrollar programas de propuestas y de reconocimiento que motiven a los empleados a generar ideas que tengan como finalidad la mejora en términos ambientales de las actividades y procesos en los que se desempeñan, y que a su vez representen ahorros para la organización por la reducción de la generación de residuos en los procesos.

\section{Estrategias financieras}

Desde la perspectiva financiera es fundamental contar con una visión de largo plazo de la gestión ambiental en las empresas, para lo que se pueden implementar las siguientes alternativas:

- Destinar eficientemente recursos hacia aspectos prioritarios relacionados con la gestión ambiental.

- Identificar las necesidades financieras del área encargada de la Gestión ambiental, así como las opciones de financiación (escenarios financieros de largo plazo) para realizar los ajustes pertinentes en el presupuesto y planear futuras inversiones.

- Proyectar a corto o mediano plazo inversiones en el área ambiental, y así darle inicio a una cultura medioambiental en la empresa.

- Mantenerse actualizados en cuanto a beneficios tributarios con los que se cuenta por un buen desempeño ambiental.
- Revisar constantemente las actualizaciones reglamentarias y de normatividad asociados a los impactos ambientales generados por la actividad económica de la empresa a fin de no incurrir en pago de multas o sanciones por incumplimiento de las mismas.

- Demostrar los efectos económicos positivos obtenidos por la gestión ambiental en la empresa y realizar una presentación escrita y en los medios de comunicación internos.

\section{Estrategias comerciales}

Se generaron estrategias que permitan atraer nuevos clientes y mejorar el porcentaje de su demanda:

- Dar a conocer a los clientes la preocupación de las empresas por el medio ambiente, ya sea por medio de cambios en la presentación de sus productos o por la publicidad.

- Incorporar a la base de datos de la organización, clientes y proveedores certificados con un manejo ambiental adecuado.

- Elaborar acuerdos estratégicos con los organismos de control ambiental en el distrito para la consecución de clientes.

- Desarrollar un plan de mercadeo, o mejorarlo según sea el caso, en el que se identifiquen clientes certificados y resaltar el manejo ambiental en el paquete de servicios ofrecidos.

- Incluir en el plan de comunicación, una presentación ante el ámbito local y para los clientes y proveedores promocionando los desarrollos tecnológicos implementados y por implementarse, las mejores prácticas aplicadas, la infraestructura con la que se cuenta y los estándares ambientales asegurados.

- Desarrollar la infraestructura y un flujo de información homogéneo para realizar el seguimiento de los clientes con exigencias medioambientales.

- Acordar con las entidades de control encargadas la entrega de información para el seguimiento comercial y planificación de actividades.

\section{Estrategias para el buen aprovechamiento de los recursos}

Para la buena utilización de los recursos se diseñaron las siguientes estrategias:

- Verificar el adecuado manejo de los recursos (energía eléctrica, agua, entre otros), apoyándose 
en los indicadores y mediciones correspondientes, con el fin de aplicar las acciones preventivas, correctivas, o de mejora necesarias.

- Asignar los recursos que sean pertinentes en respuesta de los planes de mejoramiento diseñado, medirlos y tenerlos en cuenta en el diseño de presupuestos.

- Desarrollar planes de manejo y control de los recursos.

- Estudiar la circulación y recambio y determinar los lineamientos necesarios que deberá seguir el proceso de producción.

- Identificar y cuantificar los costos en que se incurre por el mal aprovechamiento de los recursos.

\section{CONCLUSIONES}

A pesar de las ventajas competitivas que ofrece la ciudad y el mismo departamento, existe un total desconocimiento de los empresarios de aquellos beneficios productivos, competitivos y organizacionales del sistema en general, lo que genera una pérdida de la inversión realizada y ausencia en la gestión ambiental.

Actualmente existe un crecimiento dinámico en la actividad económica, el distrito de Barranquilla tiene un alto potencial para exportar si se tiene en cuenta su posición geográfica; todo este potencial hace pensar que las empresas piensen en abrir nuevos mercados y busquen nuevos métodos para lograrlos.

La implementación de la ISO 14001 ofrece muchas ventajas para la expansión de mercados nacionales e internacionales, ya que esta gestión es tan importante como la gestión de calidad por lo que se requiere la identificación de estas ventajas para su mayor aprovechamiento.

La implementación del SGA tiene mayor impacto en la gestión organizacional, seguida por el Aprovechamiento de los recursos. Los factores de mayor importancia según la percepción de los empresarios son: el desempeño jurídico, la imagen pública, las estrategias para atraer nuevos clientes, los programas de mejora en el manejo de recursos y la reducción en la generación de residuos. Por tanto, se definieron estrategias que permitan el fortalecimiento de aquellos aspectos que presentaron debilidad en la evaluación, esto con el fin de sacarle mayor provecho a la implementación del SGA.
Los SGA en las organizaciones han evolucionado de la mano a la presión social y las restricciones legales que los gobiernos han implementado, por tanto se requiere de parte del sector empresarial modelos innovadores que le apuesten a mantener estrategias preventivas para el control de la contaminación y minimización del impacto ambiental.

La sostenibilidad ambiental debe ocupar un lugar focal dentro de los objetivos misionales de las organizaciones, los indicadores de productividad y calidad no deben estar desligados de las variables de control ambiental; garantizando así mayor responsabilidad en cada uno de los eslabones de la cadena productiva.

\section{REFERENCIAS}

[1] P. Bifani. "Medio Ambiente y Desarrollo Sostenible". IEPALA Editorial. Madrid, España. 1999.

[2] D. Pearce y R. Turner. "Economía de los Recursos Naturales y del Medio Ambiente", Madrid: Colegio de Economistas de Madrid y Celeste Ediciones. 1995.

[3] PNUMA y UNDESA. "V Reunión del Consejo de Expertos de Gobierno en Consumo y Producción Sustentables para América Latina y el Caribe". UNEP. Cartagena de Indias, Colombia. 2009.

[4] M. Rodríguez y J.A. Ricart. "Dirección Medioambiental de la Empresa”. Ediciones Gestión. Barcelona, España 2000.

[5] J. Buela Fontenla. "Implantación y desarrollo de un Sistema de Gestión Ambiental en Ence-Pontevedra". Revista Galega de Economía. Vol. 11 No $^{\circ}$, pp. 1-10. 2002. ISSN: 1132-2799.

[6] C.J. Chiappeta and F.C. Almada, "Relationships between human resource dimensions and enviromental management in Companies: Proposal of a model". Journal of Cleaner Productions. Vol. 6. 2008. DOI: 10.1016/j.jclepro.2006.07.025

[7] ICONTEC. "NTC-ISO 14001. Sistemas de Gestión Ambiental. Requisitos con Orientacion para su Uso”. ICONTEC, Bogotá, Colombia. 2004.

[8] R.A. García. "Eco-eficiencia como Fuente de Ventaja Competitiva para las Pymes en México”. Instituto Politécnico Nacional 
(IPN), Mexico D.F, 2011.URL: http://tesis. ipn.mx/xmlui/handle/123456789/10277

[9] M.E. Porter. "Ser Competitivo", Ediciones Deusto. Barcelona, España, pp. 478. 2003. ISBN 84-234-2183-X

[10] A. Zutshi y A. Sohal, "Integraded management system. The experiences of three Australian Organizations". Journal of Manufacturing Technology Management. Vol. $6 \mathrm{~N}^{\circ} 2$, pp. 211-232. 2005. ISSN: 1741-038x

[11] M. Lozano and J. Valdés. "An Analysis of the Implementation of an Enviromental Management System in a Local Public Administration". Journal of Enviromental Management. Vol. 82, pp. 495-511. 2007. ISSN: 0301-4797.

[12] R. Clements. "Guía Completa de las Normas ISO 14001". España: Editorial Gestión 2000, pp. 285. 1997. ISBN: 84-8088-209-3.

[13] N.R. Escobar Moreno. "Las comunicaciones integradas de marketing (CIM) como pilar de la estrategia de marketing verde y sus implicaciones en la gestión ambiental". HYPERLINK "http://www.scielo.org.co/ scielo.php?script $=$ sci_serial $\&$ pid $=0121$ 6805\&lng=en\&nrm=iso" Revista Facultad de Ciencias Económicas: Investigación y Reflexión. Vol. 20 No 2, pp. 69-79. 2012. ISSN 0121-6805.

[14] E. Aulí. "Integración de los Factores Ambientales en las estrategias empresariales". Boletín Económico del ICE. No 800 , pp. 139148. 2002.

[15] Pro Barranquilla. "Exportaciones industriales departamento del Atlántico. Enero noviembre 2014 (US\$ millones FOB)". Barranquilla, Colombia. 2015. Recuperado 20 de Noviembre del 2014.URL: http://www. probarranquilla.org/index.php?lang=es\&op $\mathrm{c}=3 \&$ sel=2\&subsel=5, 15 de julio de 2015 .

[16] L. Bonilla. "Documento de Trabajo sobre Economía Regional”. Banco de la República, Cartagena. 2010.

[17] Cámara de Comercio de Barraquilla. "Boletín de coyuntura económica de Barranquilla". Cámara de Comercio de Barranquilla \& Fundesarrollo, pp. 10-11. Barranquilla, Colombia. 2014. Recuperado el 22 de noviembre 2014. URL: http:// www.camarabaq.org.co/wp-content/ uploads/2015/04/Boletin-Economico-deBarranquilla-enero-diciembre-2014.pdf 